\title{
Contribution of internal degree of freedom of soft molecules to Soret effect
}

\author{
Takeaki Araki and Natsumi Chikakiyo \\ Department of Physics, Kyoto University, Kyoto 606-8502, Japan
}

(Dated: May 10, 2021)

\begin{abstract}
We studied the Soret effect in binary dimer-monomer mixtures using non-equilibrium molecular dynamics simulations and investigated the pure contribution of the internal degree of freedom of flexible molecules to the Soret effect. We observed that the thermal diffusion factor tends to decrease and change its sign as the molecules become softer. We proposed two possible mechanisms of our observations: change of the molecule structures with the temperature, causing bulkier molecules to migrate to the hotter region; asymmetry of the restitution between rigid and flexible molecules, due to which flexible molecules show larger restitution when placed at the hotter region.
\end{abstract}

\section{INTRODUCTION}

When a fluid mixture is subjected to a temperature gradient, concentration gradients build up. This phenomenon is the Soret effect or thermal diffusion [1] 4 , which is widely observed in mixtures of small molecules [5, 6], polymers [7 -12], colloids [10, 13], and biomolecules [14, 15]. Since the 19 th century, many experimental, theoretical and numerical studies have been reported [1626]. However, its physical mechanisms are still not understood completely.

The Soret effect is caused by some factors; its origin is often considered the equilibrium thermodynamics [16, 22]. If the chemical potential of each species depends on the temperature, the concentration gradient is induced under the temperature gradient to homogenize this potential. The isotope effect, in which the mass difference causes the concentration gradient, is difficult to explain based on the equilibrium thermodynamics [17, 18, 23 26]. The heavier molecules tend to migrate to the cold side, while the lighter ones move to the hot side. It is also known that differences in diameter, moment of inertia, interaction between the solute and the solvent, and/or those among the solutes contribute significantly in the Soret effect [2].

When considering the Soret effect, the molecules are usually treated as rigid objects, while the internal degrees of freedom of the molecules are ignored. If flexible, the molecules are thermalized and adapt their structures to the local environment (the temperature, the pressure, and the concentration). These changes may influence the Soret effect. Polymers are examples of molecules that have large internal degrees of freedom $7-12$. If a polymer is much longer than its persistent length, it behaves as a flexible chain; it behaves as a rigid rod if it is shorter than the persistent length. It was reported that the Soret coefficient of the polymer solutions changes with the chain length when it is short. However, when it is sufficiently long, the Soret coefficient is saturated to a value that is independent of the chain length. For the polymers, the mass and the moment of inertia change with the chain length. Although some theoretical studies on the Soret effect of the polymer systems have been reported, the pure contribution of the flexibility of the molecules to the Soret effect is still unclear. The aim of this work is to investigate the roles of the internal degrees of freedom in the Soret effect by employing the simplest molecular model.

\section{MOLECULAR DYNAMICS SIMULATION}

We perform molecular dynamics simulations of mixtures of two molecular species in three dimensional rectangular boxes $\left(V=L_{x} L_{y} L_{z}\right)$. One species is the simplest molecule, which consists of a single spherical particle; the other is a dimer, which consists of two identical spherical particles. The particles in the monomers and dimers interact mutually with the Weeks-ChandlerAndersen (WCA) potential 27], which is given by

$$
U_{\mathrm{WCA}}(r)= \begin{cases}4 \epsilon\left[(\sigma / r)^{2 n}-(\sigma / r)^{n}+1 / 4\right] & \left(r \leq 2^{1 / n} \sigma\right) \\ 0 & \left(r>2^{1 / n} \sigma\right)\end{cases}
$$

where $\epsilon(=1)$ and $\sigma(=1)$ represent the strength and range of the WCA potential. $n$ is a parameter for characterizing the hardness of the WCA potential. If we do not mention explicitly, we set $n=6$. The two particles in each dimer are bounded by a harmonic potential,

$$
U_{\mathrm{sp}}(r)=k\left(r-r_{0}\right)^{2},
$$

where $k$ is the spring constant, while $r_{0}$ is the natural length of the bond, and we set $r_{0}=2^{1 / n} \sigma$. The WCA potential between the particles in each dimer is not included. The mass of the particles is given by $m$; thus the mass of the dimer is $2 \mathrm{~m}$. The numbers of the monomer and dimer molecules are given by $N_{\mathrm{m}}$ and $N_{\mathrm{d}}$. The particle packing fraction is defined by $\phi=\pi \sigma^{3} N_{\mathrm{t}} /(6 V)$, where $N_{\mathrm{t}}$ is the total particle number, $N_{\mathrm{t}}=N_{\mathrm{m}}+2 N_{\mathrm{d}}$. The mixing ratio of the dimer is given by $\chi=2 N_{\mathrm{d}} / N_{\mathrm{t}}$.

A temperature gradient is imposed along the $x$-axis by using boundary driven non-equilibrium molecular dynamics simulation. The thermostatting regions are set up at the edges $\left(x=0\right.$ and $\left.x=L_{x}\right)$ and at the center $\left(x=L_{x} / 2\right)$ of the rectangular cell, while their width is 
$0.03 L_{x}$. The temperatures in these thermostatting regions are imposed to $T=T_{\mathrm{h}}$ at the edges and $T=T_{\mathrm{c}}$ at the center $\left(T_{\mathrm{h}}>T_{\mathrm{c}}\right)$ by means of the Langevin thermostat. In the other bulk regions, the particle position and velocity are updated without any thermostat. The equations of motion are solved with velocity Verlet algorithm using LAMMPS [28], in which the time increment is $\delta t=0.0005 \sqrt{\epsilon /\left(m \sigma^{2}\right)}$. We fix the cell width as $L_{x}=80 \sigma$, while $L_{y}$ and $L_{z}$ are changed to adjust the packing fraction $\phi$. The total particle number is $N_{\mathrm{t}}=64000$, and the packing fraction is changed from $\phi=0.037$ to 0.234 . If we do not mention explicitly, we set the mixing ratio to $\chi=1 / 2$. With the SHAKE algorithm [29], we also consider dimers, in which the particle separations are fixed to $r=r_{0}$. To observe the pure effect of the dimers, we also study the Soret effect in mixtures of two monomers $\mathrm{A}$ and $\mathrm{B}$, among which the masses or the radii differ.

\section{3D SIMULATION RESULTS}

Figure 1(a) shows the profiles of the kinetic energies along the $x$-axis. The temperatures in the thermostatted regions are set to $T_{\mathrm{h}}=5 \epsilon$ and $T_{\mathrm{c}}=\epsilon$. We divide the cell into 100 slabs along the $x$-axis, and then, we calculate the local densities of the monomer particle $\rho_{\mathrm{m}}(x)$ and the dimer particle $\rho_{\mathrm{d}}(x)$, and the kinetic energies averaged per particle in each thin slab. Here, $\int \mathrm{d} x \rho_{\mathrm{d}}(x)=2 N_{\mathrm{d}}$ and $\int \mathrm{d} x \rho_{\mathrm{m}}(x)=N_{\mathrm{m}}$ are held. We plot the translational kinetic energies of the monomer $K_{\mathrm{m}}^{\text {tra }}$, and the three modes (translational $K_{\mathrm{d}}^{\mathrm{tra}}$, rotational $K_{\mathrm{d}}^{\text {rot }}$, and vibrational $K_{\mathrm{d}}^{\mathrm{vib}}$ ) of the kinetic energies of the dimer. In the equilibrium state, $K_{\mathrm{m}}^{\mathrm{tra}}, K_{\mathrm{d}}^{\mathrm{tra}}, K_{\mathrm{d}}^{\mathrm{rot}}$, and $K_{\mathrm{d}}^{\mathrm{vib}}$ should agree with $3 T / 2,3 T / 2, T$, and $T / 2$, respectively. It can be seen that all the averaged kinetic energies collapse on a master curve after appropriate scalings (see the inset of Fig. 1(a)). The kinetic energies in the thermostated regions are consistent with the target temperatures $T_{\mathrm{h}}$ and $T_{\mathrm{c}}$ and vary linearly with $x$ in the bulk regions. Thus, the temperature is controlled well in our system.

Figure 1(b) plots the local density profiles of the dimer $\rho_{\mathrm{d}}(x)$ and the monomer $\rho_{\mathrm{m}}(x)$. The spring constant is $k=400 \epsilon / \sigma^{2}$, while the packing fraction is $\phi=0.073$. Both densities are higher in the colder than in the hotter regions. It is also indicated that the density of the dimers is slightly higher than that of the monomers in the cold region. The Soret effect is induced in this mixture of dimers and monomers.

Figure 2(a) illustrates the profiles of the concentration field of the dimer in a dilute mixture, which is defined as $c(x)=\rho_{\mathrm{d}}(x) /\left(\rho_{\mathrm{m}}(x)+\rho_{\mathrm{d}}(x)\right)$. If the concentration is homogeneous, its value agrees with the mixing ratio, i.e., $c(x)=\chi$. The total packing fraction and the spring constant are set to $\phi=0.073$ and $k=400 \epsilon / \sigma^{2}$, respectively. In Fig. 2(a), we change the temperature difference $\Delta T=T_{\mathrm{h}}-T_{\mathrm{c}}$ by fixing the average temperature to $\langle T\rangle\left(=\left(T_{\mathrm{h}}+T_{\mathrm{c}}\right) / 2\right)=3 \epsilon$. In the absence of the temper-
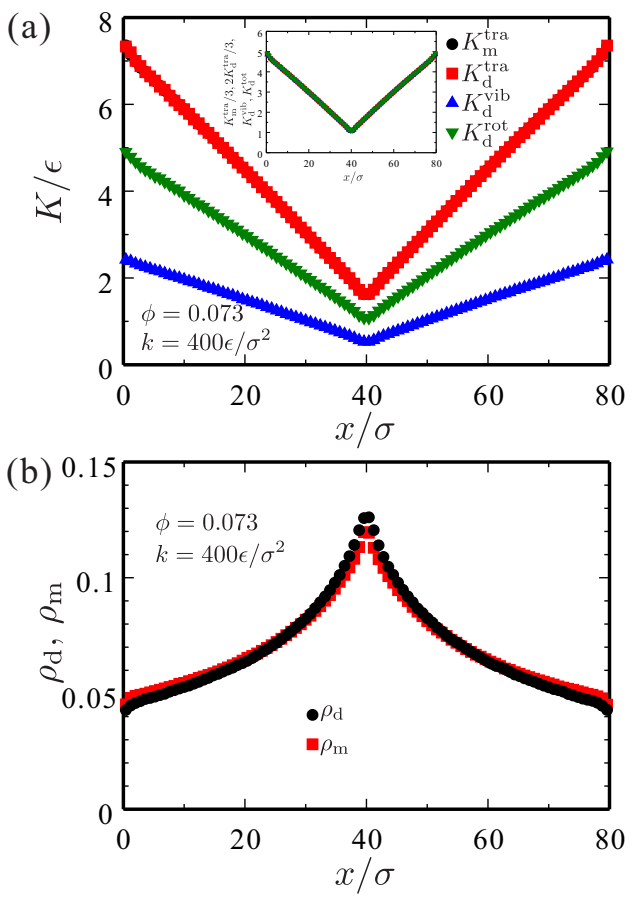

FIG. 1. (a) Profiles of the local kinetic energies of the monomers $\left(K_{\mathrm{m}}^{\text {tra }}\right)$ and the dimers $\left(K_{\mathrm{d}}^{\text {tra }}, K_{\mathrm{d}}^{\mathrm{vib}}\right.$, and $\left.K_{\mathrm{d}}^{\text {rot }}\right)$ along the temperature gradient. The average packing fraction and the spring constant are $\phi=0.073$ and $k=400 \epsilon / \sigma^{2}$, respectively. The temperatures at the thermostatted regions are $T_{\mathrm{h}}=5 \epsilon$ and $T_{\mathrm{c}}=\epsilon$. In the inset, the scaled kinetic energies are replotted. (b) Profiles of the local densities of the dimer $\rho_{\mathrm{d}}$ and the monomer $\rho_{\mathrm{m}}$. The parameters are the same as those in (a).

ature difference $(\Delta T=0)$, the concentration is almost constant in space (not shown here). As $\Delta T$ increases, the concentration near the cold region increases, while that near the hot region decreases. This means that the dimers tend to migrate to the colder side. The degree of dimer migration is almost proportional to the temperature difference. Figure 2(b) presents the profiles of the concentration in a dense mixture $(\phi=0.234)$. In contrast to the dilute mixture, the dimers migrate to the hotter region in the dense mixture. The degree of dimer migration is almost proportional to the temperature difference.

Instead of the Soret coefficient $S_{T}$, we analyze the Soret effect with the thermal diffusion factor $\alpha_{T}=T S_{T}$. We evaluate it from the local thermal diffusion factor,

$$
\alpha(x)=-\frac{T}{c(1-c)} \frac{\partial c / \partial x}{\partial T / \partial x},
$$

and average $\alpha(x)$ over the system to get $\alpha_{T}=$ $\int \mathrm{d} x \alpha(x) / L_{x}$. A positive value of $\alpha_{T}$ implies the dimer migrates to the cold side. Figure 3(a) shows the dependences of $\alpha_{T}$ against the packing fraction $\phi$. The spring constant in the dimers is changed from $k=10 \epsilon / \sigma^{2}$ to $500 \epsilon / \sigma^{2} . \alpha_{T}$ for the rigid dimer is also given in the same figure. In Fig. 3(b), we replot $\alpha_{T}$ as functions of the 

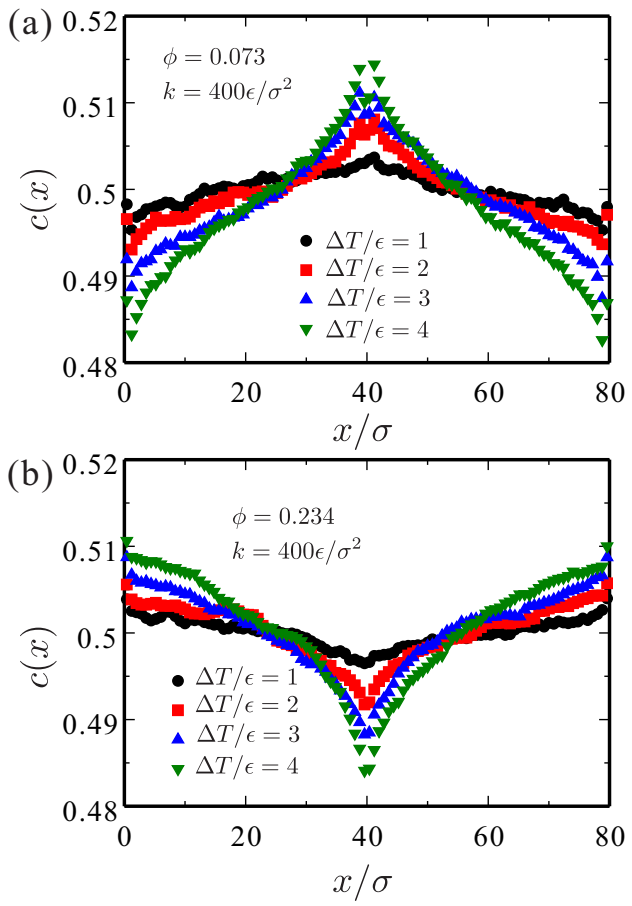

FIG. 2. (a) Profiles of the concentration of the dimers $c=\rho_{\mathrm{d}} /\left(\rho_{\mathrm{d}}+\rho_{\mathrm{m}}\right)$ in a dilute mixture of $\phi=0.073$. The temperature difference $\Delta T=T_{\mathrm{h}}-T_{\mathrm{c}}$ is changed. (b) Profiles of the concentration of the dimers in a dense mixture of $\phi=0.234$.

spring constant $k$ for several densities.

In dilute systems, the thermal diffusion factors are positive for any $k$, which indicates that the dimers migrate to the colder side, as illustrated in Fig. 2(a). As the packing fraction $\phi$ increases, the thermal diffusion factors decrease. Here the decreasing rate of $\alpha_{T}$ against $\phi$ is larger in the mixture of smaller $k$ than in that of larger $k$. For smaller $k$, the thermal diffusion factor changes its sign from positive to negative with $\phi$. In contrast, the thermal diffusion factor for the rigid dimer remains positive in the simulated range of $\phi$. The molecule becomes more rigid, and the thermal diffusion factor is likely to converge for the rigid molecules when the spring constant is increased. In Fig. 3(b), the thermal diffusion factor changes its sign with the spring constant $k$ in the intermediated mixtures $(\phi=0.146)$. Our results clearly demonstrate that the internal degrees of freedom in the molecules can influence the Soret effect, which is usually considered to be an inter-molecular phenomenon.

\section{DISCUSSION}

First, we consider the mechanism of the Soret effect in terms of the isotope effect. In Fig. 4, we plot the thermal diffusion factor in mixtures of two monomers $\mathrm{A}$ and $\mathrm{B}$ (black circles). In these mixtures, the interactions among the particles are given by Eq. (1), while
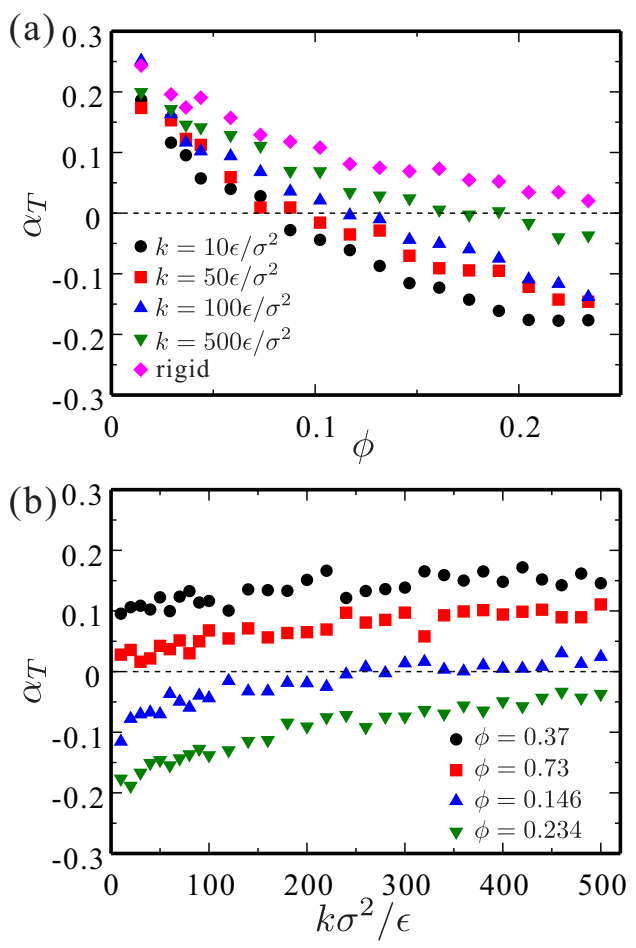

FIG. 3. (a) Plots of the thermal diffusion factor $\alpha_{T}$ against the packing fraction $\phi$. The spring constant is changed. $\alpha_{T}$ for the mixture of the rigid dimer, and monomer is also presented. (b) Plots of $\alpha_{T}$ with respect to the spring constant $k$. The packing fraction is changed.

the mass of the $\mathrm{A}$ monomer is twice larger than that of the $\mathrm{B}$ monomer, i.e., $\sigma_{\mathrm{AA}}=\sigma_{\mathrm{BB}}=\sigma_{\mathrm{AB}}=\sigma$, with $m_{\mathrm{A}}=2 m$ and $m_{\mathrm{B}}=m$. In Fig. 4 , the positive values of the thermal diffusion factor agree with the isotope effect; heavier particles A migrate to the colder region. In contrast to the decreasing thermal diffusion factor in the dimer-monomer mixtures (Fig. 3(a)), the thermal diffusion factor is increased with the packing fraction in the monomer-monomer mixtures. Thus, it is concluded that the Soret effect in the dimer-monomer mixtures is essentially different from the isotope effect. It is also known that molecules of larger inertia moments migrate to the colder region [17, 23, 24]. If the Soret effect observed in this study is due to the inertia effect, the thermal diffusion factor would be increased by using longer natural length of the bond interaction in the dimers (Eq. (2)). However, we confirmed that the thermal diffusion factor is increased when the natural length in the bond interaction is shortened (not shown here). In particular, if we set $r_{0}=0$ in Eq. (2) and use larger $k$, the thermal diffusion factor is close to that due to the isotope effect. Thus, we believe that the Soret effect in our mixtures is not due to the inertia effect.

Next we consider the contribution of the thermodynamic chemical potential to the Soret effect in our simulations. The linear non-equilibrium thermodynamic the- 


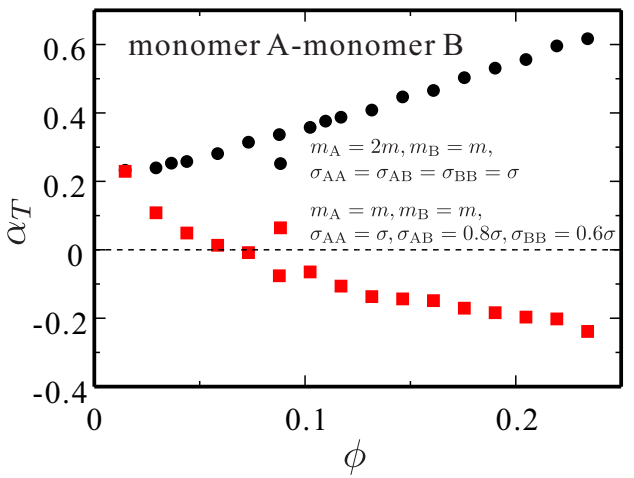

FIG. 4. The thermal diffusion factors in the mixtures of two monomers A and B are plotted against $\phi$. black circle: mixtures of monomers with different masses; red square: mixtures of monomers with different radii.

ory gives the particle flux $\vec{J}_{i}(i=\mathrm{d}$ or m) as [1]

$$
\overrightarrow{J_{i}}=-\rho_{i} L_{1 i} \nabla \frac{\tilde{\mu}_{i}}{T}+\rho_{i} L_{2 i} \nabla \frac{1}{T},
$$

where $L_{1 i}$ and $L_{2 i}$ are phenomenological kinetic coefficients, and $\tilde{\mu}_{i}$ is an effective chemical potential. In the steady state, it should vanish, i.e., $\vec{J}_{i}=0$. If $L_{2 i}=0$, the Soret effect is determined by the thermodynamic properties of the chemical potential against $\chi$ and $T$. In Figs. 5(a), (b) and (c), we represent the effective chemical potential of the dimer $\tilde{\mu}_{\mathrm{d}}$ against the mixing ratio $\chi$, the temperature $T$, and the packing fraction $\phi$, respectively. In Fig. 5(a), we changed the mixing ratio $\chi$ with fixing the total number $N_{\mathrm{t}}$. The effective chemical potential is given as $\tilde{\mu}_{\mathrm{d}}=\mu_{\mathrm{d}}-\left(v_{\mathrm{d}} / v_{\mathrm{m}}\right) \mu_{\mathrm{m}}$, where $\mu_{i}$ and $v_{i}$ are the chemical potential and partial molecular volume of the $i$-component. In Fig. 5 , we assume $v_{\mathrm{d}} / v_{\mathrm{m}}=2$, for simplicity. The chemical potentials $\mu_{\mathrm{d}}$ and $\mu_{\mathrm{m}}$ are are obtained through the Widom insertion method [30] in the simulations without the temperature difference. As indicated in Fig. 5(a), the chemical potential difference is almost independent of the mixing ratio $\chi$, while $\tilde{\mu}_{\mathrm{d}} / T$ is an increasing function of $T$ in Fig. 5(b). The chemical potential modulation induced by the temperature gradient cannot be compensated by the chemical potential change with the mixing ratio. This means that the Soret effect in our system is not caused by the thermodynamic chemical potentials, and it is purely a non-equilibrium behavior. The chemical potential difference also depends on the packing fraction in Fig. $5(\mathrm{c}) . \partial\left(\tilde{\mu}_{\mathrm{d}} / T\right) / \partial T$ is compensated roughly by $\partial\left(\tilde{\mu}_{\mathrm{d}} / T\right) / \partial \phi$ (see Fig. $\left.1(\mathrm{~b})\right)$.

As discussed above, we cannot understand our simulation results with conventional knowledge of the Soret effect, i.e., the contributions of the mass, inertia moment and chemical potential. Here, we propose possible mechanisms of the Soret effect in our dimer-monomer mixtures. In Fig. 6(a), we present the probability distribution $P(r)$ of the bond length $r$ of the dimers in the mixtures of $\phi=0.036$ and 0.234 . The spring constant is $k=10 \epsilon / \sigma^{2}$ and the temperature is changed. When
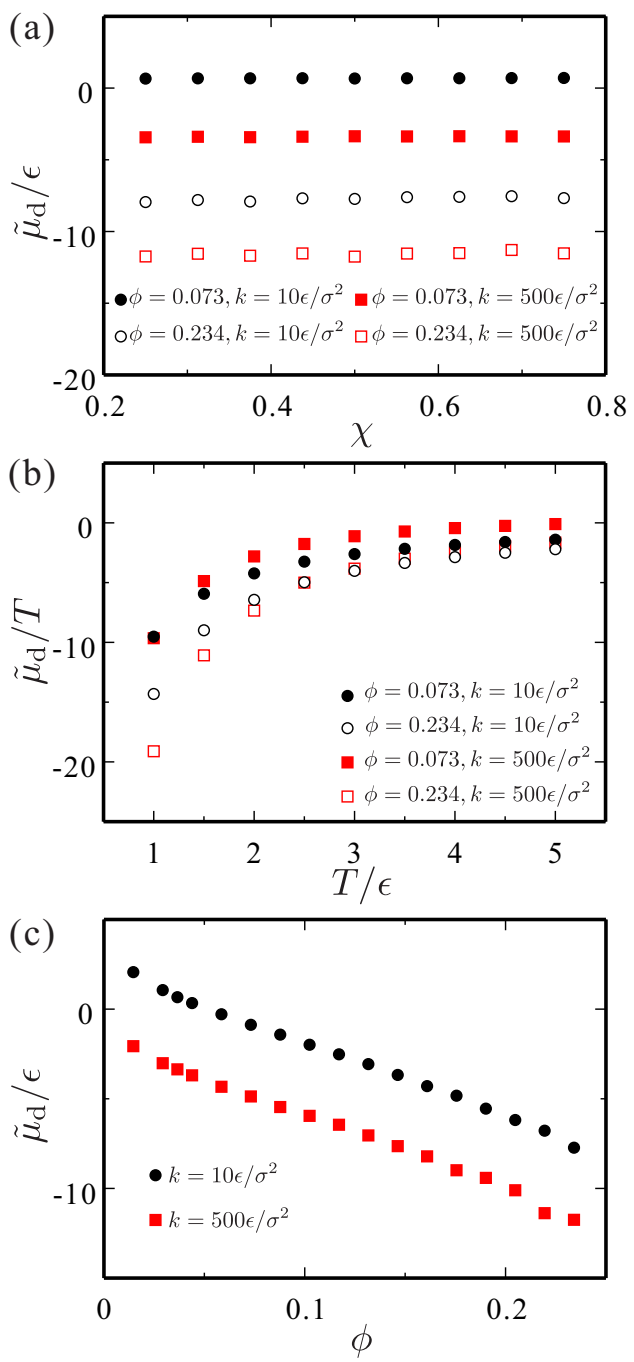

FIG. 5. The differences of the chemical potentials between the dimers and monomers $\Delta \mu$ are plotted against the temperature $\langle T\rangle$, the mixing ratio $\chi$, and the packing fraction $\phi$ in (a), (b) and (c), respectively. In (a) and (c), the temperature is $T=3 \epsilon$.

we obtain $P(r)$, the temperature gradients are not imposed. As the temperature is increased, not only the distribution width is broadened, but also the peak position is shifted to large $r$. We plot their average lengths $\langle r\rangle$ against the temperature in Fig. 6(b). Those for $k=500 \epsilon / \sigma^{2}$ are also plotted. The dimer molecule is more stretched at the high temperature. In other words, the dimer becomes bulkier. The degree of the stretching is large when $k$ is small, so that the corresponding effective volume of the dimer is increased more largely for small $k$. In the dilute mixtures $(\phi=0.036)$, the probability distribution agrees well with the Boltzmann distribution $\left(4 \pi r^{2} \times \exp \left[-U_{\mathrm{sp}}(r) / T\right]\right)$. In the dense mixtures, on the other hand, the probability distribution is inconsistent with the Boltzmann distribution. The average bond length in the dense mixture is shorter than that in the dilute mixture as shown in Fig. 6(b). The molecules are 
surrounded by other molecules, all of which are exerting non-bonded forces of the molecules. The degree of the bond stretching is suppressed more in the dense system.

In Fig. 4, we also plotted the thermal diffusion factor in other monomer-monomer mixtures as a function of the packing fraction $\phi$ (red squares). In these mixtures, the masses of both monomers are the same, while the size of the A-particle is set to be larger than that of B-particle. We replace $\sigma$ in the WCA potential (Eq. (1)) to $\sigma_{\mathrm{AA}}=\sigma, \sigma_{\mathrm{AB}}=0.8 \sigma$, and $\sigma_{\mathrm{BB}}=0.6 \sigma$ for the $\mathrm{A}-\mathrm{A}$, $\mathrm{A}-\mathrm{B}$ and $\mathrm{B}-\mathrm{B}$ pairs of the monomers, respectively. The potential strength $\epsilon$ is not changed. As the packing fraction increases, the thermal diffusion factor decreases and becomes negative eventually. The bulky A-monomers migrate to the colder region in the dilute mixtures, while they move to the hotter region in the dense mixtures. These observations are consistent with a previous study [23]. Although the physical mechanism of this Soret effect still remains unclear, we consider that the Soret effect in the dimer-monomer mixtures is related to that in these monomer-monomer mixtures. When $k$ is small, the dimer molecules become bulkier. Then the bulky dimers tend to move to the hotter region as that in the monomer-monomer mixtures, although these dimers are two times as heavy as the monomers. If $k$ is large, the dimer size does not change much, making this behavior unremarkable.

\section{1D SIMULATION}

In this section, we propose another possible mechanism of the Soret effect, with one-dimensional (1D) simulations [21]. The motions of the two particles in a dimer and a monomer particle are constrained in the $x$ axis. We prepared initial dimer and monomer, which obey the Maxwell-Boltzmann distributions at $T=T_{\mathrm{d}}$ and $T=T_{\mathrm{m}}$, respectively. The $1 \mathrm{D}$ dimer has three types of energies $\left(K_{\mathrm{d}}^{\mathrm{tra}}, K_{\mathrm{d}}^{\mathrm{vib}}\right.$, and spring potential $\left.U_{\mathrm{sp}}\right)$, the statistical averages of which are set to $T_{\mathrm{d}} / 2$. The translational kinetic energy of the monomer is set to $\left\langle K_{\mathrm{m}}^{\text {tra }}\right\rangle=T_{\mathrm{m}} / 2$. The monomer particle and one dimer particle, which we call as the first particle (and the other as the second one), interact via the WCA potential (Eq. (1)) with $n=6$. If the dimer and the monomer collide and bounce, we calculate the coefficient of restitution $e$ from the velocities of the dimer and monomers. The collision between the dimer and the monomer is inelastic, since the sum of the translational kinetic energies, $K_{\mathrm{d}}^{\text {tra }}+K_{\mathrm{m}}^{\text {tra }}$, is not conserved after the collision. In particular, when the energy due to the internal degrees of freedom $\left(K_{\mathrm{d}}^{\mathrm{vib}}+U_{\mathrm{sp}}\right)$ is transferred to the translational kinetic energies $\left(K_{\mathrm{d}}^{\text {tra }}+K_{\mathrm{m}}^{\text {tra }}\right)$, the coefficient of restitution is likely to exceed unity [31]. The coefficient of restitution is scattered statistically, so that we obtain its average with $10^{7}$ samples.

In Fig. 7(a), we present the coefficients of restitution with respect to the spring constant $k$ in two cases, $\mathrm{X}$ :
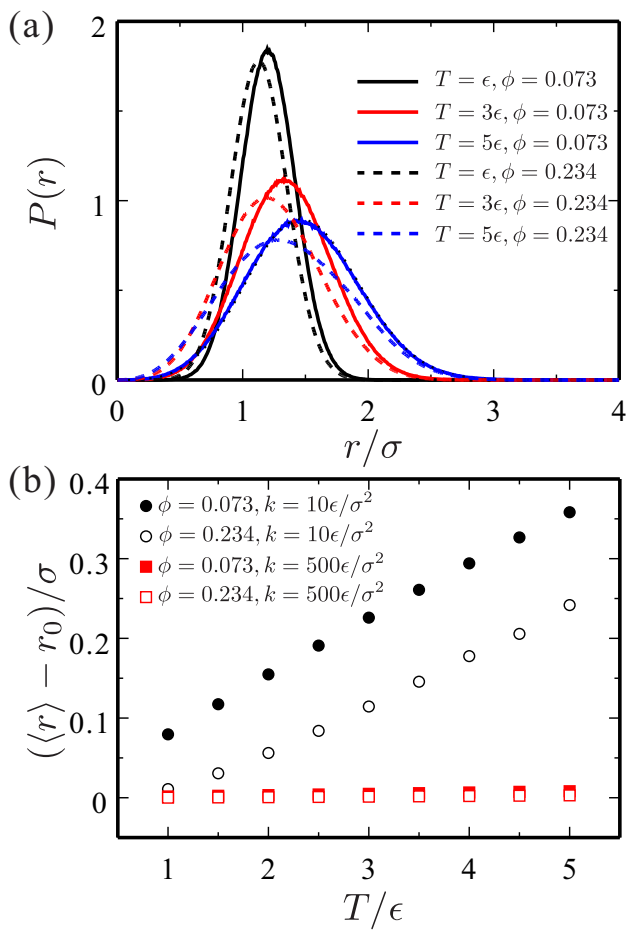

FIG. 6. (a) Probability distributions $P(r)$ of the bond length $r$ in the dimer-monomer mixtures of $\phi=0.036$ and 0.234 . The spring constant is $k=10 \epsilon / \sigma^{2}$. The temperature $T$ is changed. (b) Plots of the averaged bond length $\langle r\rangle$ with respect to the temperature $\Delta T$ in the dimer-monomer mixtures.

$\left(T_{\mathrm{d}}, T_{\mathrm{m}}\right)=(5 \epsilon, \epsilon)$, and $\mathrm{Y}:\left(T_{\mathrm{d}}, T_{\mathrm{m}}\right)=(\epsilon, 5 \epsilon)$. In both cases, the coefficient of restitution is larger than unity when $k$ is small, and it approaches to unity when $k$ is large. It can be observed that the dimer and monomer bounce asymmetrically, i.e., $e_{\mathrm{X}}>e_{\mathrm{Y}}$ over the entire range of $k$. In case $\mathrm{X}$, the coefficient of restitution is decreased monotonically to unity as the spring constant is increased. In case Y, on the other hand, the coefficient of restitution changes non-monotonically with $k$. When $k$ is small, $e_{\mathrm{Y}}$ is decreased with $k$ and becomes smaller than unity. Then, it turns to increase and approaches unity for large $k$. In case $\mathrm{X}$, the dimer is likely to have larger internal energy than the sum of the translational kinetic energies $\left(K_{\mathrm{d}}^{\mathrm{vib}}+U_{\mathrm{sp}}>K_{\mathrm{d}}^{\mathrm{tra}}+K_{\mathrm{m}}^{\mathrm{tra}}\right)$ before the collision. Upon the collision, the internal energy changes to the translational energy, increasing the coefficient of restitution. In case $\mathrm{Y}$, on the other hand, the internal energy is comparably small $\left(K_{\mathrm{d}}^{\mathrm{vib}}+U_{\mathrm{sp}}<K_{\mathrm{d}}^{\text {tra }}+K_{\mathrm{m}}^{\text {tra }}\right)$, allowing the translational energy transferred to the internal energy. As a result, the coefficient of restitution in case $\mathrm{Y}$ is likely to be smaller than that in case $\mathrm{X}, e_{\mathrm{X}}>e_{\mathrm{Y}}$. When the dimer is "hotter" than the monomer, the molecules bounce more, resulting in the negative thermal diffusion factor, with which the dimers migrate to the hotter region.

In Fig. 7(b), we plot the difference between the coefficients of restitution in two different cases, $\Delta e=e_{\mathrm{X}}-e_{\mathrm{Y}}$. 
Here, we show $\Delta e$ for several different values of $n(=$ $3,6,12$, and 24 ) in the WCA potential (Eq. (1)). In any $n, \Delta e$ is positive and it decays with $k$. With small $n$, the particles are soft, so that the collision time increase, and the monomer and the first particle in the dimer interact via the WCA potential slowly. If the intra-particle interaction is strong enough (with large $k$ ), the energy and the momentum are well transferred to the second particle in the dimer during the collision. Meanwhile, the collision becomes more elastic when $n$ is small and $k$ is large, making the coefficients of restitution $e_{\mathrm{X}}$ and $e_{\mathrm{Y}}$ converge to unity and $\Delta e$ goes to zero. When $n$ is large, on the other hand, the collision time is shortened. If the intra-molecule interaction is weak (with small $k$ ), the second particle is negligible upon the collision. Since we set the particle masses to be same, the kinetic energies and momenta are exchanged between the monomer and the first particle during the collision. However, after the collision, the translational and vibrational kinetic energies are redistributed within the dimer. If $T_{\mathrm{d}}=0$ in case $\mathrm{Y}$, in particular, the coefficient of restitution is $e_{Y}=1 / 2$ in this limit of large $n$. As a result, the difference between $e_{\mathrm{X}}$ and $e_{\mathrm{Y}}$ tends to zero more gradually when $n$ is large.
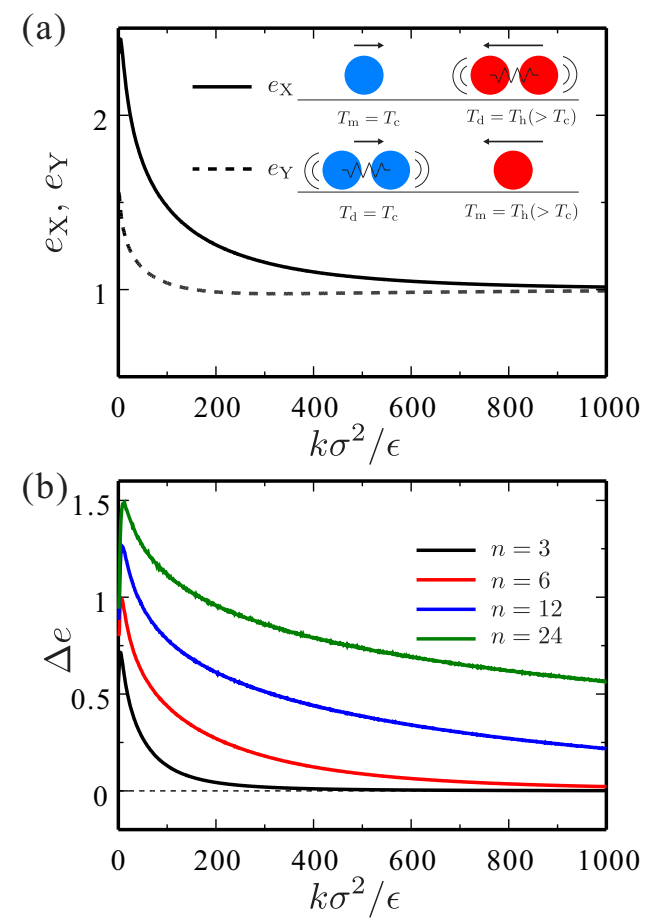

FIG. 7. (a) The coefficients of restitution between a dimer and a monomer are shown against the spring constant $k$, which are obtained in one-dimensional simulations for two cases. X: $\left(T_{\mathrm{d}}, T_{\mathrm{m}}\right)=(5 \epsilon, \epsilon), \mathrm{Y}:\left(T_{\mathrm{d}}, T_{\mathrm{m}}\right)=(\epsilon, 5 \epsilon)$. (b) The differences of the coefficients of restitution $\Delta e=e_{\mathrm{X}}-e_{\mathrm{Y}}$ is shown with the spring constant. The exponent $n$ in the WCA potential is changed.

Although we consider that this asymmetry of the restitutions can cause the Soret effect also in the actual systems, the relationship between the Soret effect in the
1D simulations and that in the above $3 \mathrm{D}$ simulations should be considered carefully. The collisions among the molecules in the 3D system are mostly oblique and not head-on. Thus, it is considered that the asymmetric restitution plays more minor roles in the three dimensional mixtures, even if it is the case. Because of the asymmetric restitution and the energy transfer, the thermalization of the internal and kinetic degrees of freedom might be violated. As shown in Fig. 1(a), however, we have not observed any relevant difference between the kinetic and vibrational temperatures. We consider that the linear temperature gradient are formed well after the particle heterogeneity due to the Soret effect is induced, although we have no evidence supporting this claim.

Here we mention a previous study of Garriga et al.21], in which the authors have considered the Soret effect in the 1D binary mixtures of light and heavy particles. They found that the asymmetric collisions lead to the strong Soret effect. Although their molecular description is quite different from ours, their findings would support our conjecture.

\section{SUMMARY}

In this article, we numerically studied the Soret effect in the dimer-monomer mixtures and found that the internal degrees of the freedom of the molecules can contribute significantly the Soret effect, which is usually treated as an inter-molecular phenomenon. In dense system, in particular, the rigid dimers migrate to the colder side, while the flexible ones move to the hotter side.

To explain the Soret effect in our system, we proposed two possible mechanisms. One regards to the changes of the molecular shape; flexible molecules adapt their volume depending on the local temperature, while bulkier dimer molecules tend to migrate to the hotter side, overcoming the isotope effect. The other regards the asymmetry of the restitutions. If a molecule having larger internal degrees of freedom is "hotter", the coefficient of restitution with the other "colder" molecule with smaller internal degrees of freedom becomes larger than that for the opposite case. To determine which of them (and/or some other mechanisms) is more dominant, we have to investigate them further through other types of test molecules. The inter-molecular interactions would be modified by the intra-structures of the molecules in the non-equilibrium conditions.

In this work, we focused on dimer molecules consisting of two particles. However, it should be noted that diatomic molecules, such as $\mathrm{O}_{2}$, are not considered with this dimer model. The vibrational modes in a real diatomic molecule are quantised and the higher modes are strongly quenched at room temperature. In this work, we aimed to consider softer molecules; polymers are appropriate candidates for such soft molecules. However, the changes of the degree of the polymerization accompany those of the mass, the moment of inertia, and the inter- 
actions, all of which influence the Soret effect. Thus, it is difficult to observe the pure effect of the flexibility on the Soret effect. It was numerically demonstrated that the difference of the chain stiffness triggers the change of the Soret effect [9]. When the degree of the polymerization is the same, the Soret coefficient of more flexible polymers, with short persistent length, tends to be smaller than those of rigid polymers with long persistent length.
We believe that our findings can help us to further understand such mysterious behaviors of the Soret effect.

\section{ACKNOWLEDGEMENTS}

This work was supported by KAKENHI (Grants No.17K05612), CREST, JST (JPMJCR1424, JPMJCR2095).
[1] S. R. de Groot and P. Mazur, Non-equilibrium Thermodynamics (Dover Publications Inc., 1985).

[2] S. Wiegand, J. Phys.: Condens. Matter 16, R357 (2004).

[3] J. K. Platten, J. Appl. Mech. 73, 5 (2005).

[4] S. Srinivasan and M. Z. Saghir, Thermodiffusion in Multicomponent Mixtures (Springer New York, 2012).

[5] R. Kita, S. Wiegand, and J. Luettmer-Strathmann, J. Chem. Phys. 121, 3874 (2004).

[6] A. Perronace, C. Leppla, F. Leroy, B. Rousseau, and S. Wiegand, J. Chem. Phys. 116, 3718 (2002).

[7] J. C. Giddings, K. D. Caldwell, and M. N. Myers, Macromolecules 9, 106 (1976).

[8] M. E. Schimpf and J. C. Giddings, J. Polym. Sci. B Polym. Phys. 27, 1317 (1989).

[9] M. Zhang and F. Müller-Plathe, J. Chem. Phys. 125, 124903 (2006).

[10] A. Würger, Phys. Rev. Lett. 98, 138301 (2007).

[11] D. Stadelmaier and W. Köhler, Macromolecules 42, 9147 (2009).

[12] A. Würger, Phys. Rev. Lett. 102, 078302 (2009).

[13] R. Piazza, Soft Matter 4, 1740 (2008)

[14] S. Duhr and D. Braun, Proc. Natl. Acad. Sci. U. S. A. 103, 19678 (2006).

[15] C. J. Wienken, P. Baaske, U. Rothbauer, D. Braun, and S. Duhr, Nat. Commun. 1, 100 (2010).

[16] E. D. Eastman, J. Am. Chem. Soc. 50, 283 (1928).

[17] P.-A. Artola, B. Rousseau, and G. Galliéro, J. Am. Chem. Soc. 130, 10963 (2008).
[18] P.-A. Artola and B. Rousseau, Mol. Phys. 111, 3394 (2013).

[19] B. Hafskjold, T. Ikeshoji, and S. K. Ratkje, Mol. Phys. 80, 1389 (1993).

[20] L. J. T. M. Kempers, J. Chem. Phys. 90, 6541 (1989).

[21] A. Garriga, J. Kurchan, and F. Ritort, J. Stat. Phys. 106, 109 (2002).

[22] A.Würger, Comptes Rendus Mécanique 341, 438 (2013).

[23] G. Galliéro, B. Duguay, J.-P. Caltagirone, and F. Montel, Fluid Ph. Equilibria 208, 171 (2003).

[24] C. Debuschewitz and W. Köhler, Phys. Rev. Lett. 87, 055901 (2001).

[25] D. Reith and F. Müller-Plathe, J. Chem. Phys. 112, 2436 (2000).

[26] S. Villain-Guillot and A. Würger, Phys. Rev. E 83, 030501(R) (2011).

[27] J. D. Weeks, D. Chandler, and H. C. Andersen, J. Chem. Phys. 54, 5237 (1971).

[28] LAMMPS molecular dynamics package WWW site: lammps.sandia.gov.

[29] D. Rapaport, The Art of Molecular Dynamics Simulation (Cambridge University Press, 2013).

[30] B. Widom, J. Chem. Phys. 39, 2808 (1963).

[31] H. Kuninaka and H. Hayakawa, Phys. Rev. E 86, 051302 (2012). 